\title{
Recent developments in intraocular lens power calculation methods-update 2020
}

\author{
Giacomo Savini ${ }^{1}$, Leonardo Taroni ${ }^{2}$, Kenneth J. Hoffer ${ }^{3,4}$ \\ ${ }^{1}$ IRCCS - G.B. Bietti Foundation, Rome, Italy; ${ }^{2}$ Ophthalmology Unit, S. Orsola-Malpighi University Hospital, University of Bologna, Bologna, \\ Italy; ${ }^{3}$ Stein Eye Institute, University of California, Los Angeles, CA, USA; ${ }^{4}$ St. Mary's Eye Center, Santa Monica, CA, USA \\ Contributions: (I) Conception and design: G Savini; (II) Administrative support: G Savini; (III) Provision of study materials or patients: None; (IV) \\ Collection and assembly of data: None; (V) Data analysis and interpretation: None; (VI) Manuscript writing: All authors; (VII) Final approval of \\ manuscript: All authors. \\ Correspondence to: Giacomo Savini, MD. IRCCS - G.B. Bietti Foundation, Via Livenza, 3 - Rome, Italy. Email: giacomo.savini@fondazionebietti.it.
}

\begin{abstract}
For many decades only a few formulas have been available to calculate the intraocular lens (IOL) power for patients undergoing cataract surgery: the Haigis, Hoffer Q, Holladay 1 and 2 and SRK/ T. In recent years, several new formulas for IOL power calculation have been introduced with the aim of improving the accuracy of refraction prediction in eyes undergoing cataract surgery. These include the Barrett Universal II, the Emmetropia Verifying Optical (EVO), the Kane, the Næser 2, the Olsen, the Panacea, the Pearl DGS, the Radial Basis Function (RBF), the T2 and the VRF formulas. Although most of them are unpublished so that their structure is unknown, we give an overview of each formula and report the results of the studies that have compared them. Their performance in short and long eyes is provided and a special focus is given on the issue of segmented axial length, which is a promising method to obtain more accurate outcomes in short and long eyes. Here, the group refractive index originally developed for the IOLMaster may not represent the best method to convert the optical path length into a physical distance. The issue of posterior and total corneal astigmatism (TCA) is discussed in relation to toric IOLs; the latest formulas for toric IOLs and their results are also reported.
\end{abstract}

Keywords: Lenses; intraocular; biometry; cataract

Submitted Mar 07, 2020. Accepted for publication Jul 24, 2020.

doi: $10.21037 / \mathrm{atm}-20-2290$

View this article at: http://dx.doi.org/10.21037/atm-20-2290

Over the past four decades, cataract surgeons had mainly used a few well-known formulas to calculate the intraocular lens (IOL) power in unoperated eyes: the Haigis (1), Hoffer Q (2), Holladay 1 (3), Holladay 2 (unpublished) and SRK/ $\mathrm{T}$ (4) formulas and they have been used on millions of eyes. Notwithstanding the rapid technological evolution of optical biometry, which saw the introduction of a number of new devices (5-10), little advancement has been observed for IOL formulas, until about 10 years ago, when several new formulas for unoperated eyes have been developed, particularly in the last 5 years (11-13). These formulas were designed to improve the accuracy of refractive predictions, which are still far from being perfect, even in normal unoperated eyes. The effort of researchers has led to the publication of some landmark papers with thousands of eyes, whose measurements have been used to compare the refractive accuracy of such formulas.

At the same time, new formulas for toric IOLs have been developed. The purpose of this review is to give an update on the best methods to calculate the IOL power in unoperated eyes with and without astigmatism.

\section{New formulas}

* Barrett Universal II formula (BUII): actually, this is not a totally new formula, but the evolution of the Barrett Universal I, which was published by Graham Barrett, MD, in 1987 as a thick-lens paraxial formula 
$(14,15)$. The BUII is essentially based on the same concept, although the author subsequently introduced several modifications over the years. These included using data to predict the radius of the globe as alluded to in the original article rather than an empirical method. Later on, lens thickness (LT) and corneal diameter (CD) were added. The name changed into BUII in 2013. The formula is unpublished, so that little is known about its structure. Although Koch et al. listed it among vergence formulas (16), the BUII is based on ray tracing (Graham Barrett, personal communication, 2019). It is available for free at https://calc.apacrs.org/barrett_universal2105/ (accessed on February 16 $6^{\text {th }} 2020$ ) and uses axial length $(\mathrm{AL})$, keratometry $(\mathrm{K})$, anterior chamber depth (ACD, measured from epithelium to lens) to predict the IOL position; LT and CD can be entered optionally. In several studies it was ranked among the most accurate formulas.

* Emmetropia Verifying Optical (EVO) Formula: this is a thick-lens formula (unpublished) developed by Tun Kuan Yeo, MD, of Singapore. Version 2.0 is available for free at www.evoiolcalculator.com (accessed on February $16^{\text {th }} 2020$ ) and uses AL, K, and ACD as the predictors (LT and central corneal thickness (CCT) are optional). Version 1.0 has been tested by a few studies, which reported its high accuracy.

* Kane Formula: this is another unpublished formula, which was developed by Jack X Kane, MD. According to its author, it is based on theoretical optics and contains some elements of artificial intelligence, but its structure is largely unknown. The formula is available for free at www.iolformula.com (accessed on February $16^{\text {th }} 2020$ ) and uses AL, K, ACD, and gender to predict the IOL position, with LT and CCT being optional factors. An increasing number of studies have reported excellent outcomes with this formula.

* Ladas Super Formula: this method was originally developed by John G Ladas, MD, et al. as a combination of the Hoffer Q, Holladay 1, Holladay 2 (with Wang-Koch adjustment for AL adjustment) and SRK/T formulas $(12,17)$. Based on a threedimensional model, this method was developed to choose the best formula for each eye. In 2019, the formula was revised using the postoperative data of more than 4,000 eyes and is now based on artificial intelligence (Ladas Super Formula AI), available at www.iolcalc.com (accessed on July $11^{\text {th }}, 2020$ ).
* Næser 2 formula: this is a thick-lens formula developed by Kristian Næser, MD. The original Næser 1 formula was based on the manufacturer's cutting-card for the IOL anterior and posterior curvature (18). The Næser 2 uses calculated data for the architecture of the IOL. AL measurements are optimized so that the refractive outcomes are equally good in short, medium and long eyes. The results reported by the author were as good as those obtained with the BUII formula (19).

* Olsen Formula: this formula has undergone several refinements over the years. It was first described as early as 1987 (20) and was then refined in subsequent years $(21,22)$, until the most recent version, which is based on ray-tracing and the $\mathrm{C}$ constant concept (23). The latter estimates the IOL position solely on the preoperative measurements of ACD and LT. The formula can be downloaded at www. phacooptics.net (accessed on February 16 ${ }^{\text {th }} 2020$ ). Interestingly, the PhacoOptics software offers two options to predict the position of the IOL: by default, four predictors are used for this purpose (AL, K, ACD and LT), as in the earlier versions of this formula; however, $\mathrm{AL}$ and $\mathrm{K}$ can be omitted from the prediction, so that the $\mathrm{C}$ constant approach is adopted. For this reason, two versions of the Olsen formula are described in the literature: the former is the 4-factor version, also known as $\mathrm{Olsen}_{\text {standalone, the latter, based }}$ on the $\mathrm{C}$ constant, is the 2 -factor version and is the one installed on optical biometers. The refractive accuracy has been reported to be high for both.

* Panacea: this (unpublished) formula was developed by David Flickier, MD, and is available for free at www.panaceaiolandtoriccalculator.com (accessed on February $\left.16^{\text {th }} 2020\right)$. It is the only formula enabling surgeons to enter the corneal asphericity (Q-value) and the ratio between the anterior and posterior corneal curvature, which should improve the refractive accuracy. The results have not yet been published in large studies.

* Pearl DGS: this formula has been developed by a team of French ophthalmologists (G. Debellemanière, D. Gatinel, and A. Saad, and hence its name), who relied on artificial intelligence. It is available for free at www. iolsolver.com (accessed on February 16 ${ }^{\text {th }} 2020$ ). The formula is unpublished so that nothing is known of its structure. Since it was introduced in 2019, no study has yet reported its outcomes. 
* RBF Calculator (Radial Basis Function): launched in 2016, this was the first IOL power calculation method based purely on artificial intelligence. It is available for free at www.rbfcalculator.com (accessed on February $\left.16^{\text {th }} 2020\right)$ and is installed on the Lenstar (HaagStreit, Switzerland). Version 2.0 is based on more than 12,000 eyes and, opposite of the original version, can calculate IOL power for a target different than zero. Required inputs are AL, K and ACD (LT, CCT and $\mathrm{CD}$ are optional). Several papers have shown that it leads to good outcomes, although it has not been ranked as the best in any study.

* T2 Formula: this a modification of the original SRK/ T, described by Richard M. Sheard, MD, et al. in 2010 (11). The main purpose of the authors was to correct a non-physiological behavior of the corneal height prediction (so-called "cusp phenomenon") (24). It has been shown to be more accurate than the SRK/T.

* VRF formula: this vergence formula, developed by Oleksiy Voytsekhivskyy, MD, is one of the few published ones (25). The IOL position is predicted from AL, K, ACD and CD. Compared to standard vergence formulas, the results reported by the author are good, but it still needs to be validated against other modern formulas.

\section{Formulas comparisons}

Several studies recently compared the above-described formulas as well as older formulas. They reported similar outcomes, as in most cases the best formulas were the BUII and Olsen, followed by the Haigis. When newer formulas were analyzed, some of these (e.g., Kane and EVO) provided equally excellent outcomes.

In 2016, Kane et al. compared the results of different formulas in 3,241 eyes measured with the IOLMaster 500 (Carl Zeiss Meditec, Germany) (26). Since this biometer was not able to measure LT, the Olsen formula could not be investigated. Kane's study was one of the first to demonstrate that the BUII was more accurate than traditional formulas, including the Haigis and Holladay 2. It displayed a lower mean absolute error (MAE) and median absolute error (MedAE) as well as a higher percentage of eyes with a prediction error (PE) lower than \pm 0.50 diopters (D). Such advantage was evident in medium, mediumlong and long eyes, but not in short eyes. It is interesting to note that the results were good, but not excellent, notwithstanding constant optimization and use of just one
IOL model. The BUII achieved, in fact, only $72.3 \%$ of eyes with a $P E$ within $\pm 0.50 \mathrm{D}$, a percentage lower than the corresponding value reported by other studies. This may be due to the inclusion of data from patients operated on by different surgeons and refracted by different examiners.

Just a few months earlier, Cooke and Cooke reported similar outcomes in 1,079 eyes, with the advantage of having measurements from both IOLMaster and Lenstar, so that the Olsen formula could be included (27). The BUII ranked first with the IOLMaster and second with Lenstar, whereas

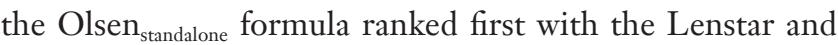
achieved the highest percentage of eyes with a PE within $\pm 0.50 \mathrm{D}(83.7 \%)$. The Haigis formula was the third best. This was the first paper to report better results with the Olsen formula available on PhacoOptics software compared to the Olsen formula pre-installed on the Lenstar.

In 2017, Kane et al. further contributed to our knowledge by comparing the BUII to three new formulas in 3,211 eyes: the FullMonte method, the Ladas Super Formula and the RBF (27). They found that none of the newer formulas gave more accurate results over the BUII; they also underperformed traditional formulas, such as the Holladay 1 and SRK/T. The BUII ranked first in two additional papers with small sample size and multifocal IOLs $(28,29)$.

In 2018, Melles et al. published their study with the largest database, using 13,301 eyes with the Acrysof SN60WF (Alcon Laboratories, Inc., TX, USA) and 5,200 eyes with the Acrysof SA60AT being analyzed (30). Their findings closely mirror those previously reported $(28,31)$, as the BUII and Olsen formulas ranked first and second, followed by the Haigis formula. In addition to the refractive outcomes, this paper reported some interesting relationships, such as the dependence of the SRK/T on K, the dependence of the Hoffer Q on ACD and the lack of correlation between the PE of the BUII, Haigis and Olsen and AL. However, it did not include newer formulas and for this reason an update was published in 2019 (32). Here the most accurate outcomes were achieved by the Kane, BUII, Olsen and EVO 1.0 formulas, which all achieved at least $80 \%$ of eyes with a $\mathrm{PE}$ within $\pm 0.50 \mathrm{D}$; the $\mathrm{RBF}$ was less accurate than the best formulas, but still better than traditional vergence formulas.

In 2020, Darcy et al. reported the analysis of 10,930 eyes implanted with 4 different IOL models (33). Preoperative biometry was performed by means of partial coherence interferometry (PCI), so that LT was not available. They found that the Kane was the most accurate formula; they also reported that RBF 2.0 improved over the 1.0 (it 
ranked second) and that the Holladay 2 formula with $\mathrm{AL}$ adjustment improved over the original Holladay 2 formula. Overall, the percentage of eyes with a PE within $\pm 0.50 \mathrm{D}$ was still relatively low (between $70 \%$ and $72 \%$ ) (also true for the best performing formulas).

In 2020 another important study was published. Savini et al. reported the results of 15 formulas in a smaller sample $(\mathrm{n}=150)$ of eyes, all with the same IOL model (Acrysof SN60WF) (8). Preoperative biometry was performed with an optical biometer based on swept-source optical coherence tomography (OA-2000, Tomey, Japan). They confirmed the high accuracy of the BUII, EVO 1.0, Holladay with $\mathrm{AL}$ adjustment, Kane, RBF 2.0 and Olsen $_{\text {standalone }}$ formulas, which achieved at least $88 \%$ of eyes with a $\mathrm{PE}$ within $\pm 0.50 \mathrm{D}$. They also reported good outcomes for the older formulas (Haigis, Hoffer Q, Holladay 1 and SRK/T), which had between $85.33 \%$ and $84.67 \%$ of eyes with a PE within $\pm 0.50 \mathrm{D}$. Finally, they highlighted that today it is possible to have high percentages $(>55 \%)$ of eyes with a PE within $\pm 0.25 \mathrm{D}$ and this should become one of the main outcomes to be reported in future studies. These results are similar to those reported by the same group with different optical biometers, the Aladdin (Topcon EU, Italy) and the Galilei G6 (Ziemer, Switzerland) (34,35).

\section{Subgroup analysis: short and long eyes}

The refractive accuracy of IOL power calculation in short eyes ( $\mathrm{AL}<22 \mathrm{~mm}$ ) is still lower than in medium length eyes. Since 1993, it has been well accepted that the Hoffer Q formula performed best in eyes shorter than $22 \mathrm{~mm}$, but recently no formula has been shown to be superior compared to the others. Shrivastava et al. did not find statistically significant differences in 50 eyes among the BUII, Haigis, Hoffer Q, Holladay 2 and RBF 1.0 (36). Similar outcomes were reported by Göcke et al., when comparing the same formulas plus the Olsen formulas in 86 eyes (37), as well as by Kane et al. in the subgroup of short eyes from their 2016 paper (31). Unfortunately, Melles did not statistically analyze formulas accuracy for short eyes (30). Overall, these studies show that the percentage of short eyes with a PE within $\pm 0.50 \mathrm{D}$ is lower, i.e., between $50 \%$ and $70 \%$ of eyes according to the different studies. Caution therefore is required when selecting the IOL power in these eyes and it might be wise to use formulas that take preoperative ACD into account when dealing with eyes that have a shallow ( $<2.40 \mathrm{~mm}$ ) ACD (38). This suggestion is valid also in eyes with medium AL and ACD of $3.00 \mathrm{~mm}$ or less or ACD of $3.5 \mathrm{~mm}$ or more (39).

The situation is better in long eyes ( $\mathrm{AL}>26 \mathrm{~mm}$ ), where modern formulas led to remarkable improvements over the past. Formulas whose accuracy is not influenced by AL should be preferred. The SRK/T is still a valid option on condition that specifically optimized constants are used (40). Published papers reported the good performance of the BUII $(26,30,31,33,41,42)$, Kane $(32,33)$, Olsen $(26,33,42)$, and RBF $2.0(33,41)$ formulas. The Haigis formula is accurate for long eyes (31), but it does not seem the best option when the AL is $>30 \mathrm{~mm}$, as better outcomes have been achieved in these eyes with the BUII and Olsen formulas (41). Overall, around $75 \%$ of eyes may be expected to have a PE within $\pm 0.50 \mathrm{D}$ with the best formulas (42).

\section{Axial length adjustments}

The issue of AL adjustment was discussed in 2009 by Fam and $\operatorname{Lim}$ (43), who proposed a transformation of the AL and $\mathrm{K}$ measurements of the IOLMaster to improve the refractive outcomes in eyes with extreme AL. Subsequently, it gained wide popularity thanks to Wang et al. with the socalled Wang/Koch formula (16). In order to reduce the risk of postoperative hyperopia in eyes with $\mathrm{AL}>25.0 \mathrm{~mm}$ and achieve a zero mean $\mathrm{PE}$, they generated some regression formulas to optimize the AL (measured by PCI with the IOLMaster) for the Haigis, Hoffer Q, Holladay 1 and SRK/T formulas. These equations shortened the measured $\mathrm{AL}$. The reason why AL should be shortened in long eyes was explained by the authors: when optical biometry was developed, the optical path length measured by PCI had to be converted into a geometrical distance matching the AL measured by immersion ultrasound biometry (which was the gold standard at that time) (1). Since PCI cannot measure LT, it was not possible to use the specific refractive index of each intraocular dioptric medium and a "group refractive index" was used. This represents an average refractive index, which works correctly in eyes with medium AL, but is not accurate in long eyes, where a higher percentage of vitreous is present. As a consequence, the group refractive index overestimates AL. Subsequent studies have shown that the original Wang/Koch AL adjustment not only avoids postoperative hyperopia, but induces, on average, a myopic outcome $(30,41)$. The authors therefore modified the original formula and made it less aggressive in the reduction of AL (44). A subsequent paper confirmed that the modified Wang/Koch formula induces less myopia (41). The modified Wang/Koch formula should be applied to the 
Holladay 1 formula in eyes longer than $26.5 \mathrm{~mm}$ and to the SRK/T formula in eyes longer than $27.0 \mathrm{~mm}$ (it should not be applied to the Haigis formula). The optimized constants available on the User Group for Laser Interference Biometry (ULIB) website should be used for all IOLs but the Acrysof MA60MA, for which the manufacturer's constant should be used.

However, based on a database of more than 18,000 eyes, they further refined the AL adjustment separately for the Holladay 1 and Holladay 2 formulas. These adjustments rely on nonlinear regression analysis and should be applied to eyes longer than $24.0 \mathrm{~mm}$ (45). They are available on the Holladay Consultant software as "Holladay adjustment" and represent the main innovation of the Holladay 2 formula, recently tested by different studies $(8,33)$.

Other authors questioned the validity of traditional AL measurements by optical biometry and investigated the so-called "sum-of-segments AL", which is obtained by adding the length of each segment (CCT, ACD, LT and vitreous). Cooke and Cooke were able to generate the sumof-segment AL from Lenstar measurements and compare it to the AL shown on the printout, which is obtained with the group refractive index (46). They found that in both short and long eyes the sum-of-segments AL improved the accuracy of Hoffer Q, Holladay 1, Holladay 2 and SRK/ $\mathrm{T}$ formulas but worsened the performance of the BUII and Olsen formulas. Similar outcomes have been reported by Wang et al. using segmented AL rather than AL calculated with a single group refractive index (47). Since sum-ofsegments $\mathrm{AL}$ is not available on most optical biometers (the only exception is the Argos by MOVU, Japan), an easy way to approximate it would be beneficial. Therefore, the same authors developed the Cooke-modified AL (CMAL), a regression formula which improves the PE of the Hoffer Q, Holladay 1, Holladay 2 and SRK/T formulas (48).

\section{Calculation of toric IOLs}

For more than 10 years the attention of researchers has been drawn by posterior corneal astigmatism (PCA). In 2009, Ho et al. were the first to compare keratometric astigmatism (KA) and total corneal astigmatism (TCA) (49). Using a rotating Scheimpflug camera (Pentacam, Oculus), they found that $10.3 \%$ of the candidates for toric IOLs had either a KA magnitude that differed by $>0.50 \mathrm{D}$ from the TCA magnitude or a KA angle that differed by $>10^{\circ}$ from the TCA angle. In 2011, Koch et al. used a dual
Scheimpflug analyzer (Galilei, Ziemer) to observe that PCA shows, in more than $85 \%$ of the eyes, a vertically aligned steeper meridian. Given the negative power of the posterior corneal surface, which causes it to behave with regard to refractive astigmatism exactly opposite to the anterior corneal surface, the steeper the curvature of a given posterior corneal meridian, the more negative the power. Therefore, the steep vertical meridian in the posterior surface produces an against-the-rule (ATR) refractive astigmatism $(50,51)$. As a consequence, according to vector analysis KA leads to an estimation error of TCA of $0.22 \mathrm{D}$ @ $180^{\circ}$, on average. Similar outcomes were subsequently reported by other authors, who confirmed that on average KA overestimates TCA in eyes with WTR astigmatism and underestimates it in eyes with ATR astigmatism (52-54). Not surprisingly, when comparing KA to TCA, the latter was found to predict more accurately the postoperative refractive astigmatism in eyes receiving toric and non-toric IOLs $(55,56)$. Without a doubt, the ideal situation would be that we could determine with a direct measurement the PCA and calculate the TCA in this way. However, this has proven to be a formidable challenge for today's technology. Currently notwithstanding improvements in corneal imaging (Scheimpflug tomographers, anterior segment optical coherence tomographers), there is not really a gold standard for the direct determination of the PCA in a given eye, and in fact, it has been demonstrated that using measured TCA is less accurate than using predicted TCA (57-62).

The first method used to predict TCA was the Baylor toric IOL nomogram, published in 2013, which took into account the mean values of PCA that they found (ATR astigmatism) and aimed to leave eyes after the toric IOL implantation with small amounts of WTR refractive astigmatism. It was required to manually perform the calculation following the guidelines indicated in some tables (63). Shortly after the Barrett Toric Calculator was released. This calculator applies a mathematical algorithm, also based fundamentally on the ATR refractive effect of PCA, to indirectly deduce posterior corneal radii, and is linked to the Barrett Universal II formula. By entering patient data, power is automatically obtained for both the spherical equivalent and the toricity of the IOL. This is available for free on the website of the APACRS (https:// calc.apacrs.org/toric_calculator20/Toric\%20Calculator. aspx, accessed on February $\left.26^{\text {th }}, 2020\right)$. Other methods were then developed by Abulafia et al. (64), Savini and Næser (58), 
Goggin et al. (65), and Holladay et al. (66), as well as by some IOL manufacturers (67). Other recently introduced toric calculators are those developed by Kane (https://www. iolformula.com, accessed on July $\left.11^{\text {th }}, 2020\right)$ and on the website of the EVO formula (www.evoiolcalculator.com, accessed on July $\left.11^{\text {th }}, 2020\right)$. The Abulafia-Koch calculator is used to calculate the toricity of the IOL manufactured by Physiol (https://www.physioltoric.eu/physioltoric, accessed on February $26^{\text {th }}, 2020$ ); the Næser/Savini toric calculator is available for free at https://www.soiweb.com/ toric-calculator (accessed on February 26 ${ }^{\text {th }}, 2020$ ); the Holladay toric calculator is available for free at https:// www.hic-soap.com/calc/preop (accessed on February $27^{\text {th }}$, 2020). Overall, these methods are considered today the gold standards to calculate toric IOLs. Their higher accuracy with respect to measured TCA may depend on the fact that direct determinations are still not accurate enough and in addition, prediction can take other factors into account (not only PCA): one of these may be IOL tilting, which has been shown to induce ATR refractive astigmatism (68).

On average, the mean error in the prediction of refractive astigmatism (also known as "error in refractive astigmatism" or "centroid error in predicted residual astigmatism") is close to zero, because estimating methods perform an optimization of corneal astigmatism to reach this goal. However, since when obtaining the vectorial mean value of the astigmatisms, those with different directions tend to cancel each other, these almost perfect values must be analyzed with caution. Indeed, a clinically relevant standard deviation can be observed in all studies, so that the percentage of eyes within $\pm 0.50 \mathrm{D}$ of the predicted refractive astigmatism is still relatively low and ranges between $55 \%$ and $78 \%(57-59,69)$. Further studies and new technological developments are required to improve the direct evaluation of TCA, and therefore the calculation of the power of toric intraocular lenses.

\section{Intraoperative aberrometry}

Intraoperative aberrometry is a relatively new technology used to calculate both the sphere and cylinder of the IOL to be implanted. The most common system is the Optiwave Refractive Analysis (ORA, Alcon), which is attached to the microscope and can measure aphakic and pseudophakic refraction during cataract surgery. Usually, the refraction is taken after cataract removal, when the capsular bag is filled with a viscoelastic. In unoperated eyes, studies have shown that the refractive outcomes of IOL power calculation are similar to those of optical biometry $(70,71)$.

\section{Acknowledgments}

Funding: This work was supported by the Italian Ministry of Health and Fondazione Roma.

\section{Footnote}

Provenance and Peer Review: This article was commissioned by the Guest Editor (Dr. Andrzej Grzybowski) for the series "Recent developments in cataract surgery" published in Annals of Translational Medicine. The article was sent for external peer review organized by the Guest Editor and the editorial office.

Conflicts of Interest: All authors have completed the ICMJE uniform disclosure form (available at http:// dx.doi.org/10.21037/atm-20-2290). The series "Recent developments in cataract surgery" was commissioned by the editorial office without any funding or sponsorship. GS reports grants from Italian Ministry of Health, grants from Fondazione Roma, during the conduct of the study; personal fees from Alcon, personal fees from CSO, personal fees from Oculus, personal fees from Zeiss, outside the submitted work. KJH reports personal fees from Carl Zeiss Meditec, personal fees from Haag-Streit, personal fees from Oculus, personal fees from Ziemer, personal fees from Topcon EU/Visia Imaging, personal fees from Tomey, personal fees from Movu, personal fees from Heidelberg Engineering, outside the submitted work. The authors have no other conflicts of interest to declare.

Ethical Statement: The authors are accountable for all aspects of the work in ensuring that questions related to the accuracy or integrity of any part of the work are appropriately investigated and resolved.

Open Access Statement: This is an Open Access article distributed in accordance with the Creative Commons Attribution-NonCommercial-NoDerivs 4.0 International License (CC BY-NC-ND 4.0), which permits the noncommercial replication and distribution of the article with the strict proviso that no changes or edits are made and the original work is properly cited (including links to both the formal publication through the relevant DOI and the license). 
See: https://creativecommons.org/licenses/by-nc-nd/4.0/.

\section{References}

1. Haigis W, Lege B, Miller N, et al. Comparison of immersion ultrasound biometry and partial coherence interferometry for intraocular lens calculation according to Haigis. Graefes Arch Clin Exp Ophthalmol 2000;238:765-73.

2. Hoffer KJ. The Hoffer Q formula: a comparison of theoretic and regression formulas. J Cataract Refract Surg 1993;19:700-712; errata, 1994;20:677;2007;33:2-3.

3. Holladay JT, Prager TC, Chandler TY, et al. A three-part system for refining intraocular lens power calculations. J Cataract Refract Surg 1988;14:17-24.

4. Retzlaff JA, Sanders DR, Kraff MC. Development of the SRK/T intraocular lens power calculation formula. J Cataract Refract Surg 1990;16:333-340; erratum, 528.

5. Ortiz A, Galvis V, Tello A, et al. Comparison of three optical biometers: IOLMaster 500, Lenstar LS 900 and Aladdin. Int Ophthalmol 2019;39:1809-18.

6. Hoffer KJ, Shammas HJ, Savini G, et al. Multicenter study of optical low-coherence interferometry and partialcoherence interferometry optical biometers with patients from the United States and China. J Cataract Refract Surg 2016;42:62-7.

7. Kanclerz P, Hoffer KJ, Rozema JJ, et al. Repeatability and reproducibility of optical biometry implemented in a new optical coherence tomographer and comparison with a optical low-coherence reflectometer. J Cataract Refract Surg 2019;45:1619-24.

8. Savini G, Hoffer KJ, Balducci N, et al. Comparison of formula accuracy for intraocular lens power calculation based on measurements by a swept-source optical coherence tomography optical biometer. J Cataract Refract Surg 2020;46:27-33.

9. Kurian M, Negalur N, Das S, et al. Biometry with a new swept-source optical coherence tomography biometer: repeatability and agreement with an optical lowcoherence reflectometry device. J Cataract Refract Surg 2016;42:577-81.

10. Yang CM, Lim DH, Kim HJ, et al. Comparison of two swept-source optical coherence tomography biometers and a partial coherence interferometer. PLoS One 2019;14:e223114.

11. Sheard RM, Smith GT, Cooke DL. Improving the prediction accuracy of the SRK/T formula: the T2 formula. J Cataract Refract Surg 2010;36:1829-34.
12. Ladas JG, Siddiqui AA, Devgan U, et al. A 3-D "Super Surface" Combining Modern Intraocular Lens Formulas to Generate a "Super Formula" and Maximize Accuracy. JAMA Ophthalmol 2015;133:1431-6.

13. Hoffer KJ, Savini G. Clinical Results of the Hoffer H-5 Formula in 2707 Eyes: First 5th-generation Formula Based on Gender and Race. Int Ophthalmol Clin 2017;57:213-9.

14. Barrett GD. Intraocular lens calculation formulas for new intraocular lens implants. J Cataract Refract Surg 1987;13:389-96.

15. Barrett GD. An improved universal theoretical formula for intraocular lens power prediction. J Cataract Refract Surg 1993;19:713-20.

16. Koch DD, Hill W, Abulafia A, et al. Pursuing perfection in intraocular lens calculations: I. Logical approach for classifying IOL calculation formulas. J Cataract Refract Surg 2017;43:717-8.

17. Wang L, Shirayama M, Ma XJ, et al. Optimizing intraocular lens power calculations in eyes with axial lengths above $25 \mathrm{~mm}$. J Cataract Refract Surg 2011;37:2018-27.

18. Naeser K. Intraocular lens power formula based on vergence calculation and lens design. J Cataract Refract Surg 1997;23:1200-7.

19. Næser K, Savini G. Accuracy of thick-lens intraocular lens power calculation based on cutting-curd or calculated data for lens archirtecture. J Cataract Refract Surg 2019;45:1422-9.

20. Olsen T. Theoretical approach to intraocular lens calculation using Gaussian optics. J Cataract Refract Surg 1987;13:141-5.

21. Olsen T, Corydon L, Gimbel H. Intraocular lens power calculation with an improved anterior chamber depth prediction algorithm. J Cataract Refract Surg 1995;21:313-9.

22. Olsen T. Prediction of the effective postoperative (intraocular lens) anterior chamber depth. J Cataract Refract Surg 2006;32:419-24.

23. Olsen T, Hoffmann P. C constant: new concept for ray tracing-assisted intraocular lens power calculation. J Cataract Refract Surg 2014;40:764-73.

24. Haigis W. Occurrence of erroneous anterior chamber depth in the SRK/T formula. J Cataract Refract Surg 1993;19:442-6.

25. Voytsekhivskyy OV. Development and clinical accuracy of a new intraocular lens power formula (VRF) compared to other formulas. Am J Ophthalmol 2018;185:56-67.

26. Cooke DL, Cooke TL. Comparison of 9 intraocular 
lens power calculation formulas. J Cataract Refract Surg 2016;42:1157-64.

27. Kane JX, Van Heerden A, Atik A, et al. Accuracy of 3 new methods for intraocular lens power selection. J Cataract Refract Surg 2017;43:333-9.

28. Shajari M, Kolb CM, Petermann K, et al. Comparison of 9 modern intraocular lens power calculation formulas for a quadrifocal intraocular lens. J Cataract Refract Surg 2018;44:942-8.

29. Reitblat O, Assia EI, Kleinmann G, et al. Accuracy of predicted refraction with multifocal intraocular lenses using two biometry measurement devices and multiple intraocular lens power calculation formulas. Clin Exp Ophthalmol 2015;43:328-34.

30. Melles RB, Holladay JT, Chang WJ. Accuracy of intraocular lens calculation formulas. Ophthalmology 2018;125:169-78.

31. Kane JX, Van Heerden A, Atik A, et al. Intraocular lens power formula accuracy: comparison of 7 formulas. J Cataract Refract Surg 2016;42:1490-500.

32. Melles RB, Kane JX, Olsen T, et al. Update on intraocular lens calculation formulas. Ophthalmology 2019;126:1334-5.

33. Darcy K, Gunn D, Tavassoli S, et al. Assessment of the accuracy of new and updated intraocular lens power calculation formulas in 10930 eyes from the UK National Health Service. J Cataract Refract Surg 2020;46:2-7.

34. Savini G, Hoffer KJ, Barboni P, et al. Accuracy of optical biometry combined with Placido disc corneal topography for intraocular lens power calculation. PLoS One 2017;12:e0172634.

35. Savini G, Negishi K, Hoffer KJ, et al. Refractive outcomes of intraocular lens power calculation using different corneal power measurements with a new optical biometer. J Cataract Refract Surg 2018;44:701-8.

36. Shrivastava AK, Behera P, Kumar B, et al. Precision of intraocular lens power prediction in eyes shorter than 22 $\mathrm{mm}$ : an analysis of 6 formulas. J Cataract Refract Surg 2018;44:1317-20.

37. Gökce SE, Zeiter JH, Weikert MP, et al. Intraocular lens power calculations in short eyes using 7 formulas. J Cataract Refract Surg 2017;43:892-7.

38. Eom Y, Kang SY, Song JS, et al. Comparison of Hoffer Q and Haigis formulae for intraocular lens power calculation according to the anterior chamber depth in short eyes. Am J Ophthalmol 2014;157:818-24.e2.

39. Gökce SE, Montes De Oca I, Cooke DL, et al. Accuracy of 8 intraocular lens calculation formulas in relation to anterior chamber depth in patients with normal axial lengths. J Cataract Refract Surg 2018;44:362-8.

40. Petermeier K, Gekeler F, Messias A, et al. Intraocular lens power calculation and optimized constants for highly myopic eyes. J Cataract Refract Surg 2009;35:1575-81.

41. Liu J, Wang L, Chai F, et al. Comparison of intraocular lens power calculation formulas in Chinese eyes with axial myopia. J Cataract Refract Surg 2019;45:725-31.

42. Rong X, He W, Zhu Q, et al. Intraocular lens power calculation in eyes with extreme myopia: comparison of Barrett Universal II, Haigis, and Olsen formulas. J Cataract Refract Surg 2019;45:732-7.

43. Fam HB, Lim KL. Improving refractive outcomes at extreme axial lengths with the IOLMaster: the optical axial length and keratometric transformation. Br J Ophthalmol 2009;93:678-83.

44. Wang L, Koch DD. Modified axial length adjustment formulas in long eyes. J Cataract Refract Surg 2018;44:1396-7.

45. Wang L, Holladay JT, Koch DD. Wang/Koch axial length adjustment for the Holladay 2 formula in long eyes. J Cataract Refract Surg 2018;44:1291-1292. Errata: J Cataract Refract Surg 2019;45:117.

46. Cooke DL, Cooke TL. A comparison of two methods to calculate axial length. J Cataract Refract Surg 2019;45:284-92.

47. Wang L, Cao D, Weikert MP, et al. Calculation of axial length using a single group refractive index versus using different refractive indices for each ocular segment: theoretical study and refractive outcomes. Ophthalmology 2019;126:663-70.

48. Cooke DL, Cooke TL. Approximating sum-of-segments axial length from a traditional optical low-coherence reflectometry measurement. J Cataract Refract Surg 2019;45:351-4.

49. Ho JD, Tsai CY, Liou SW. Accuracy of corneal astigmatism estimation by neglecting the posterior corneal surface measurement. Am J Ophthalmol 2009;147:788-95.

50. Koch DD, Ali SF, Weikert MP, et al. Contribution of posterior corneal astigmatism to total corneal astigmatism. J Cataract Refract Surg 2012;38:2080-8.

51. Koch DD. The enigmatic cornea and intraocular lens calculations: the LXXIII Edward Jackson Memorial Lecture. Am J Ophthalmol 2016;171:xv-xxx

52. Tonn B, Klaproth OK, Kohnen T. Anterior surface-based keratometry compared with Scheimpflug tomographybased total corneal astigmatism. Invest Ophthalmol Vis Sci 2014;56:291-8. 
53. Savini G, Versaci F, Vestri G, et al. Influence of posterior corneal astigmatism on total corneal astigmatism in eyes with moderate to high astigmatism. J Cataract Refract Surg 2014;40:1645-53.

54. Galvis V, Tello A, Niño CA, et al. Total corneal astigmatism and posterior corneal surface. J Refract Surg 2015;31:423.

55. Savini G, Næser K. An analysis of the factors influencing the residual refractive astigmatism after cataract surgery with toric intraocular lenses. Invest Ophthalmol Vis Sci 2015;56:827-35.

56. Klijn S, Reus NJ, Van Der Sommen CM, et al. Accuracy of total corneal astigmatism measurements with a Scheimpflug imager and a color light-emitted diode corneal topographer. Am J Ophthalmol 2016;167:72-8.

57. Abulafia A, Hill WE, Franchina M, et al. Comparison of methods to predict residual astigmatism after intraocular lens implantation. J Refract Surg 2015;31:699-707.

58. Savini G, Næser K, Schiano-Lomoriello D, et al. Optimized keratometry and total corneal astigmatism for toric intraocular lens calculation. J Cataract Refract Surg 2017;43:1140-8.

59. Ferreira TB, Ribeiro P, Ribeiro FJ, et al. Comparison of methodologies using estimated or measured values of total corneal astigmatism for toric intraocular lens power calculation. J Refract Surg 2017;33:794-800.

60. Galvis V, Tello A, Niño CA, et al. Total corneal astigmatism measurement precision. Invest Ophthalmol Vis Sci 2015;56:5912.

61. Koch DD. The posterior cornea: hiding in plain sight. Ophthalmology 2015;122:1070-1.

62. Shajari M, Sonntag R, Ramsauer M, et al. Evaluation of total corneal power measurements with a new optical biometer. J Cataract Refract Surg 2020;46:675-681.

Cite this article as: Savini G, Taroni L, Hoffer KJ. Recent developments in intraocular lens power calculation methodsupdate 2020. Ann Transl Med 2020;8(22):1553. doi: 10.21037/ atm-20-2290
63. Koch DD, Jenkins RB, Weikert MP, et al. Correcting astigmatism with toric intraocular lenses: effect of posterior corneal astigmatism. J Cataract Refract Surg 2013;39:1803-9.

64. Abulafia A, Koch DD, Wang L, et al. New regression formula for toric intraocular lens calculations. J Cataract Refract Surg 2016;42:663-71.

65. Goggin M, Zamora-Alejo K, Esterman A, et al. Adjustment of anterior corneal astigmatism values to incorporate the likely effect of posterior corneal curvature for toric intraocular lens calculation. J Refract Surg 2015;31:98-102.

66. Holladay JT, Pettit G. Improving toric intraocular lens calculations using total surgically induced astigmatism for a $2.5 \mathrm{~mm}$ temporal incision. J Cataract Refract Surg 2019;45:272-83.

67. Canovas C, Alarcon A, Rosén R, et al. New algorithm for toric intraocular lens power calculation considering the posterior corneal curvature. J Cataract Refract Surg 2018;44:168-74.

68. Weikert MP, Golla A, Wang L. Astigmatism induced by intraocular lens tilt evaluated via ray tracing. J Cataract Refract Surg 2018;44:745-9.

69. Abulafia A, Barrett GD, Kleinmann G, et al. Prediction of refractive outcomes with toric intraocular lens implantation. J Cataract Refract Surg 2015;41:936-44.

70. Raufi N, James C, Kuo A, et al. Intraoperative aberrometry vs modern preoperative formulas in predicting intraocular lens power. J Cataract Refract Surg 2020;46:857-61.

71. Zhang Z, Thomas LW, Leu SY, et al. Refractive outcomes of intraoperative wavefront aberrometry versus optical biometry alone for intraocular lens power calculation. Indian J Ophthalmol 2017;65:813-7. 